Review Article

\title{
Implications on the Therapeutic Potential of Statins via Modulation of Autophagy
}

\author{
Armita Mahdavi Gorabi, ${ }^{1}$ Nasim Kiaie, ${ }^{1}$ Saeed Aslani, ${ }^{2}$ Thozhukat Sathyapalan, ${ }^{3}$ \\ Tannaz Jamialahmadi, ${ }^{4,5}$ and Amirhossein Sahebkar ${ }^{6}{ }^{6,7,8}$ \\ ${ }^{1}$ Research Center for Advanced Technologies in Cardiovascular Medicine, Tehran Heart Center, Tehran University of \\ Medical Sciences, Tehran, Iran \\ ${ }^{2}$ Department of Immunology, School of Medicine, Tehran University of Medical Sciences, Tehran, Iran \\ ${ }^{3}$ Department of Academic Diabetes, Endocrinology and Metabolism, Hull York Medical School, University of Hull, Hull, UK \\ ${ }^{4}$ Department of Food Science and Technology, Quchan Branch, Islamic Azad University, Quchan, Iran \\ ${ }^{5}$ Department of Nutrition, Faculty of Medicine, Mashhad University of Medical Sciences, Mashhad, Iran \\ ${ }^{6}$ Applied Biomedical Research Center, Mashhad University of Medical Sciences, Mashhad, Iran \\ ${ }^{7}$ Biotechnology Research Center, Pharmaceutical Technology Institute, Mashhad University of Medical Sciences, Mashhad, Iran \\ ${ }^{8}$ School of Pharmacy, Mashhad University of Medical Sciences, Mashhad, Iran
}

Correspondence should be addressed to Amirhossein Sahebkar; amir_saheb2000@yahoo.com

Armita Mahdavi Gorabi and Nasim Kiaie contributed equally to this work.

Received 19 May 2021; Accepted 20 July 2021; Published 31 July 2021

Academic Editor: Juan Gambini

Copyright (C) 2021 Armita Mahdavi Gorabi et al. This is an open access article distributed under the Creative Commons Attribution License, which permits unrestricted use, distribution, and reproduction in any medium, provided the original work is properly cited.

Statins, which are functionally known as 3-hydroxy-3-methyl-glutaryl-CoA (HMG-CoA) inhibitors, are lipid-lowering compounds widely prescribed in patients with cardiovascular diseases (CVD). Several biological and therapeutic functions have been attributed to statins, including neuroprotection, antioxidation, anti-inflammation, and anticancer effects. Pharmacological characteristics of statins have been attributed to their involvement in the modulation of several cellular signaling pathways. Over the past few years, the therapeutic role of statins has partially been attributed to the induction of autophagy, which is critical in maintaining cellular homeostasis and accounts for the removal of unfavorable cells or specific organelles within cells. Dysregulated mechanisms of the autophagy pathway have been attributed to the etiopathogenesis of various disorders, including neurodegenerative disorders, malignancies, infections, and even aging. Autophagy functions as a double-edged sword during tumor metastasis. On the one hand, it plays a role in inhibiting metastasis through restricting necrosis of tumor cells, suppressing the infiltration of the inflammatory cell to the tumor niche, and generating the release of mediators that induce potent immune responses against tumor cells. On the other hand, autophagy has also been associated with promoting tumor metastasis. Several anticancer medications which are aimed at inducing autophagy in the tumor cells are related to statins. This review article discusses the implications of statins in the induction of autophagy and, hence, the treatment of various disorders.

\section{Introduction}

Autophagy is crucial for maintaining the homeostasis of cells, both in physiological and pathological conditions $[1,2]$. In the normal state, autophagy is involved in the degradation and clearance of the nonfunctional or aged cells and cell organelles that are potentially hazardous for cell survival [3, 4]. Cells need to maintain the balance between cell death and survival to modulate normal physiology and maintain homeostasis [5]. Nonetheless, if the cell is provided with limited amounts of nutrients, autophagy of the unnecessary organelles confers a limitation of energy demands, ensuring cell survival [6]. Dysregulated autophagy in molecular levels has been associated with the etiology and pathogenesis of various disorders, including autoimmunity [7], malignancy $[8]$, and neurodegenerative diseases $[9,10]$. Autophagy 
functions as a double-edged sword during tumor metastasis. On the one hand, it plays a role in inhibiting metastasis through restricting necrosis of tumor cells, suppressing the infiltration of the inflammatory cell to tumor niche, and developing the release of mediators that induce potent immune responses against tumor cells. On the other hand, autophagy has also been associated with promoting tumor metastasis [11]. Studies have revealed that autophagy promotes drug resistance in ovarian cancer cells, leading to tumor cell survival $[12,13]$. On the contrary, inhibition of apoptosis has been associated with increased toxicity of cancer drugs against tumor cells [14]. In addition to cancer, the impairment of autophagy interferes with the clearance of amyloid-beta, leading to the development of Alzheimer's disease (AD) [15]. Consequently, the modulation of autophagy has been on track recently as a therapeutic strategy in treating neurodegenerative disorders [16].

Statins pharmacologically are inhibitors of the 3hydroxy-3-methyl-glutaryl-CoA (HMG-CoA) reductase that primarily decrease low-density lipoprotein-cholesterol levels (LDL-C) and triglyceride. For a long time, statins have been prescribed for patients with higher levels of cholesterol, LDL-C, and hypertriglyceridemia in patients with cardiovascular disorders and diabetes $[17,18]$. However, the current line of evidence has shown that statins have numerous lipid-independent (pleiotropic) actions [19-26]. Among the pleiotropic effects of statins is modulation of autophagy in various cells, providing a promising therapeutic strategy in treating disorders with impaired autophagy as primary underlying pathogenesis $[27,28]$.

In this review, we focus on the molecular pathways of autophagy and those modified by statins and try to discuss the implications of statins in the therapy of disorders related to the regulation of autophagy.

\section{Autophagy in Depth}

If autophagy mechanisms could not reduce stress levels and reverse the cell injury in the organelles, apoptosisassociated cell death occurs [29], called ferroptosis [30]. Several stress-related factors, such as a limited level of nutrients and cellular energy, increased rate of reactive oxygen species (ROS), and accumulation of aggregated and misfolded proteins, may trigger autophagy [31, 32]. Autophagy is manifested in three ways: microautophagy, macroautophagy, and chaperone-mediated autophagy [32, 33]. Macroautophagy, the primary form of autophagy, is responsible for the degradation of most proportion of the cytoplasmic cargos [34]. Autophagy-related genes (Atgs) are involved in the function of the macroautophagy, which is functionally involved in the degradation of cytoplasmic components in lysosomes, maturation of the phagosome, and exocytosis [35]. Microautophagy, considered the nonselective lysosomal degradative, is mediated through the direct surrounding of a cytoplasmic cargo via autophagic tubes. Microautophagy of soluble substrates in the cell is commonly stimulated by nitrogen starvation or rapamycin that activates signaling pathways. The significant microautophagy roles are maintaining the size of organelles, homeostasis of the cell membrane, and modulat- ing the cell survival during nitrogen deprivation [36]. Like heat-shock proteins (HSPs), several chaperones are involved in recognising aggregated or misfolded proteins and directing them towards lysosomes for degradation or disaggregation [37]. In case chaperons could not resolve aggregated or misfolded proteins, autophagy-related cell death occurs [38].

After being discovered in 1963, autophagy signaling pathways have much been studied in the context of several human diseases $[39,40]$. The four stages of autophagy include nucleation, elongation, maturation, and degradation [41]. At the first stage, translocation of the Unc-51 like autophagy activating kinase 1 (ULK1) initiation complex to the early autophagosome (or phagophore) occurs. Inhibition of the mammalian target of rapamycin (mTOR) leads to the release of the ULK1 complex, promoting the activation and translocation of the complex. In the second stage, the class III phosphatidyl triiodide (PI3) kinase complex facilitates the elongation of the phagophore membrane. Next, two autophagy receptors, including p62/sequestosome-1 and optineurin, promote the cargo's recruitment to the expanding phagophore. These two receptors are ligated with microtubule-associated protein light chain 3- (LC3-) II on the expanding phagophore through LC3-interacting regions (LIRs) and with polyubiquitin chains on autophagy substrates through the ubiquitin-like (Ubl) domains. At the third step, the maturation of the phagophore occurred by fusion of the phagophore with lysosome upon the recruitment of substrate and its enclosure. Finally, at the fourth step, the fusion of the autophagosome with lysosome results in the degradation of the autophagic substrates via acid hydrolases located in the lysosome. Degradation causes the recycling of nutrients inside the phagolysosomes to the cell cytosol [42].

The critical regulator of autophagy is $\operatorname{mTOR}[43,44]$. During the standard physiological settings with no stress and enough energy, mTOR signaling interrupts the ULK1 and PI3 kinase/vacuolar protein sorting protein 34 (Vps34) complex, resulting in the inhibition of autophagy. ULK1 and PI3 kinase/Vps34 complex are critical in composing the autophagosome structure [45]. During stress situations, mTOR signaling is modulated, resulting in activation of the ULK1 and PI3K/Vps43 complex. Beclin-1 is also involved in the development of autophagosomes $[46,47]$. The adenosine monophosphate-activated protein kinase (AMPK) is activated during the limited energy available to the cells. AMPK triggers the ULK1 complex, which activates Vps34 and Beclin-1, leading to the constitution of the autophagosome [48]. Moreover, AMPK stimulates the elongation stage, followed by the function of LC3 and ATG5-ATG12 [49]. During the fusion of the autophagosome with a lysosome in the final stage, several proteins, including synaptosomeassociated protein 29 (SNAP29), Syntaxin 17 (STX17), vesicle-associated membrane protein 8 (VAMP8), homotypic fusion and protein sorting-tethering complex (HOPS), LC3/gamma-aminobutyric acid receptor-associated protein (GABARAP), and Rab7, are involved [50, 51]. In the energy-deprived state, inhibition of mitogen-activated protein kinase (MAPK) results in inhibition of mTOR phosphorylation and hence activation of autophagy $[52,53]$. 


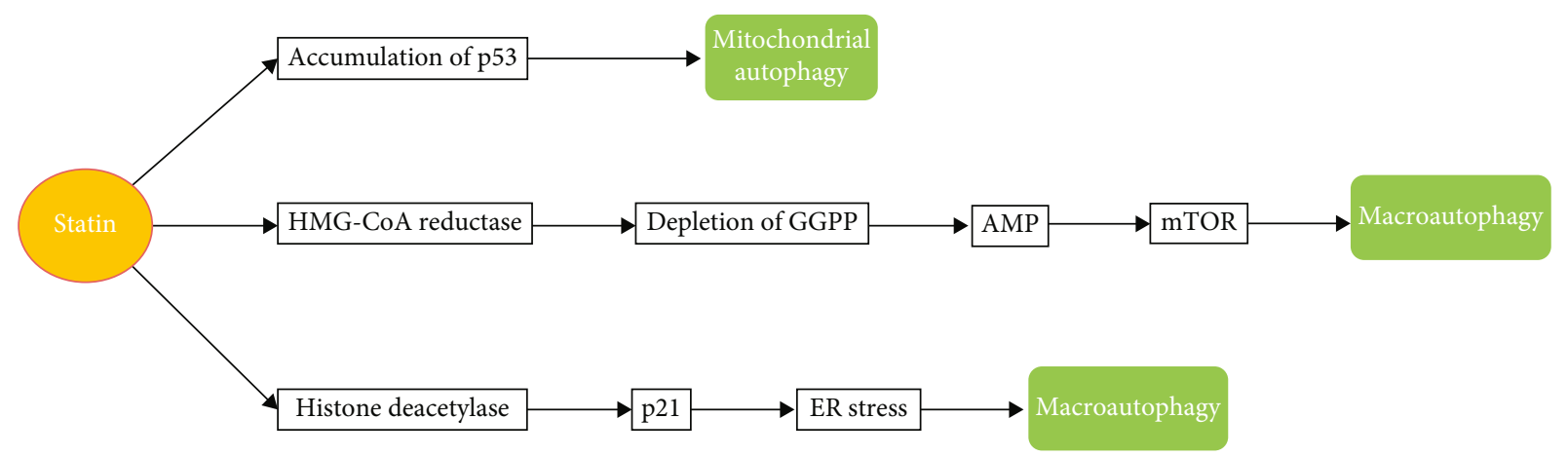

FIGURE 1: Mechanisms by which statins promote autophagy.

\section{Statins}

Statins are plant-derived chemicals that inhibit the HMGCoA, therefore lowering the levels of LDL [54-56]. By inhibition of HMG-CoA, statins interfere with the mevalonate (MVA) pathway or the HMG-CoA reductase pathway, leading to reduced generation of LDL cholesterol and development of autophagy (Figure 1) [57, 58]. The first statins identified were mevastatin (mevinolin) extracted from Penicillium citrinum [59]. Next, lovastatin was isolated from Aspergillus terreus and got approval by the Food and Drug Administration in 1987 [60]. That notwithstanding, due to adverse effects during trials, cerivastatin was banned. Subsequently, several compounds of the statin family were identified, including pravastatin, simvastatin, fluvastatin, and atorvastatin.

A novel functional pathway of statin had recently been described by Schonewille et al. According to their investigations, statins could promote the excretion of cholesterol through the fecal route [61]. Nonetheless, statins probably act through perturbation of the MVA pathway and reduce the serum cholesterol level [62]. Both farnesyl diphosphate (FPP) and geranylgeranyl diphosphate (GGPP), which are generated through the MVA pathway, are critical in the proper functioning of GTPase proteins, such as Ras, Rac, and Rho [62-65]. By regulating such GTPase proteins, statins may affect cancer cells and confer a therapeutic pathway for treating various malignancies [66]. Additionally, statins lower the LDL-C level, which correlates with a reduced risk of CVD $[67,68]$. Over the past few decades, the prescription of statins has been increased globally and will continue to do so in the upcoming decades [69]. Consequently, further trials need to be implemented to characterize the safety and adverse effects of statins.

The potential impact of statins in reducing diabetes mellitus (DM) has also been evaluated. According to the observations, long-term or first-time use of statins was not associated with DM incidence [70]. Nonetheless, a recent study on short-term consumption of statins (in comparison to those that have never used statins) showed that it might promote susceptibility to the development of DM [71]. Hence, the rate of DM might increase after the widespread use of statin, which requires attention. Despite several adverse effects, statins effectively lower lipid and treatment of CVD according to the outcomes by the meta-analysis of clinical trials [72, 73]. The beneficial characteristics of statins are not confined to lowering lipids and improvement of dyslipidemia. Statins also have several properties that potentiate them in the respective therapy of different disorders, including antiinflammatory $[74,75]$, antioxidant $[76,77]$, neuroprotective $[78,79]$, and antitumor [80]. Consumption of statins has also been related to a decreased rate of Alzheimer's disease and the development of dementia [81]. Additionally, the longtime use of statins has been shown to promote the survival of cancer patients, suggesting the potential of statins in the treatment of tumors [82].

One of the limiting points of statins is their low bioavailability. For example, simvastatin has the lowest bioavailability of $5 \%$, and pravastatin, rosuvastatin, and atorvastatin have bioavailability ranging from 10 to $20 \%$ [83]. That notwithstanding, attempts have been developed to promote the bioavailability of statins. As an example, nanocarriers have been tried to increase the bioavailability of statins [84].

\section{Antitumor Effects of Statins}

Despite an increase in the incidence of malignancies, the mortality rate has been reduced because of progression in the development of therapeutic strategies over the past few years [85]. Regarding the critical role of autophagy in the pathogenesis of cancers, modulation of autophagy might be a promising therapeutic approach. Studies have revealed that simvastatin stimulates the ERK1/2 and Akt pathways and therefore dysregulates autophagy flux and promotes cancer cell death [86]. Lovastatin has been used as a complementary agent in the treatment of malignancies. For example, lovastatin has been used as an adjutant in cisplatin-based therapy for the treatment of cancers. Lovastatin therapy promotes the levels of LC3B-II, a marker for autophagosomes, leading to upmodulation in the autophagic cell death and reduced viability of the tumor cells in malignant pleural mesothelioma [87]. Through triggering the autophagy pathway via Rac/phospholipase C/inositol 1,4,5-triphosphate axis, it was seen that lovastatin significantly reduced the migration and survival of the human malignant peripheral nerve sheath tumor cell lines ST88-14, STS-26T, and NF90-8 [88]. Stimulation of autophagic cell death excels the apoptotic cell death during tumor therapy. This is because of the resistance of cancer 
cells to death by apoptosis and the stimulatory properties of the apoptosis-bearing tumor cells on the proliferation and vitality of the cells in the vicinity. Additionally, the combination treatment of tumor cells with farnesyltransferase inhibitor and lovastatin resulted in efficient autophagy of the tumor cells [88]. It was shown that simvastatin suppressed the nuclear factor- $\kappa \mathrm{B}$ (NF- $\kappa \mathrm{B})$ in the human gastric cancer cells and decreased the proliferation of these cells. Furthermore, simvastatin increased the sensitivity of gastric tumor cells to the chemotherapy compound capecitabine [89]. Hence, statins might indirectly confer antitumor effects by increasing chemosensitivity in the tumor cells and increasing chemotherapy's efficacy. Therefore, statins can be used in combination with chemotherapy to obtain better outcomes.

During the progression of tumor cells, angiogenesis plays a critical role. Several therapeutic strategies have been developed to inhibit angiogenesis pathways to suppress tumor development $[90,91]$. It was shown that atorvastatin promoted autophagy in human umbilical vein endothelial cells and inhibited angiogenesis. Additionally, atorvastatin promoted the expression of LC3II and decreased the survival and proliferation of malignant cells [92]. Furthermore, pitavastatin induced autophagy in the melanoma cells by promoting the LC3II levels [93]. Rosuvastatin was shown to stimulate autophagy in papillary thyroid carcinoma. It was demonstrated that rosuvastatin decreased cell viability and caused a G1 phase arrest in the papillary thyroid cell line. Hence, rosuvastatin may be an alternative treatment for refractory papillary thyroid cancer [94]. Studies support the antitumor effect of fluvastatin by promoting autophagy. It was suggested that fluvastatin developed autophagy by upmodulation of the expression of the autophagy-related gene (ATG) 5 and ATG7 and therefore repressed the metastasis of lung adenocarcinoma to bone tissue [95]. Additionally, fluvastatin increased LC3II levels and induced autophagy, reducing proliferation and survival of lymphoma cells in vitro [95]. Therefore, the antitumor effects of statins may be due to suppressing the angiogenesis in the tumor tissue, above and beyond the direct effect on the tumor cells themselves.

\section{Osteoarthritis Therapy}

Osteoarthritis (OA) is defined as a bone and joint disorder characterized by progressive degradation of cartilage [96]. The role of autophagy in the modulation of chondrocytes and etiopathogenesis of OA has been demonstrated [80]. On the other side, reports show the beneficial therapeutic characteristics of statins (like simvastatin) in patients with OA. However, an inflammatory state might suppress autophagy pathways and exacerbate the OA [97]. It was observed that simvastatin resulted in the downregulation of matrix metalloproteinases (MMPs) and promoted autophagy in OA patients. Simvastatin developed the autophagy pathway by suppressing the phosphorylation of mTOR and upmodulating LC3 [97]. It has been shown that pravastatin also modulates autophagy in rat articular chondrocytes [98] and ameliorates OA patients. As a promising therapeutic strategy, the administration of pravastatin in patients with OA might promote autophagy, which results in the deceleration of cartilage degradation. It was demonstrated that pravastatin therapy in the endothelial progenitor cells led to MAPK signaling inhibition, which in turn resulted in activation of autophagy through promoting the transcription of expression of Beclin1, LC3II, ATG7, and ATG12. Pravastatin also inhibited mTOR and activated MAPK signaling, leading to the induction of autophagy and thereby causing improvement in the vascular necrosis in the femoral head [99]. Recently, a metaanalysis by Wang et al. indicated that statin administration might not be associated with a lower risk of incidence and progression of OA. Nonetheless, an opposite function of atorvastatin and rosuvastatin was observed in OA [100]. Hence, the potential beneficial effect of statins on OA improvement is a matter of debate, but it might underlie the suppression of autophagy in the chondrocytes by several statins.

\section{Neuroprotective Effects of Statins}

Among the significant neurodegenerative disorders with a considerable rate of mortality are Parkinson's disease (PD), multiple sclerosis (MS), amyotrophic lateral sclerosis (ALS), and $\mathrm{AD}$. Evidence has associated the etiopathogenesis of neurodegenerative disorders to aging as well as impairments in the autophagy pathways $[101,102]$. In patients with ALS, there is a motor neuron death, and simvastatin has been shown to suppress the GGPP synthesis, develop autophagic vacuoles, and decrease LC3II/I levels, which results in reduced autophagy in the neurons and ameliorates disease manifestations [103]. Rosuvastatin was shown to provide therapeutic neuroprotective effects in cerebral ischemic/reperfusion injury [104]. Moreover, it was revealed that the combination treatment of rats (adult male Sprague-Dawley rats receiving middle cerebral artery occlusion surgery as an animal model of cerebral ischemia/reperfusion injury) by rosuvastatin and resveratrol could efficiently reduce the activation of caspase- 3 and serum levels of interleukin- (IL-) $1 \beta$. Besides, it promoted the expression of Beclin-1 and LC3II and increased the ratios of Bcl-2/Bax and LC3II/LC3I, leading to autophagy perturbation. These events reduced the cerebral infarct volume and the neurologic defective score, leading to ameliorating disease outcomes [104]. In vitro investigations have demonstrated the neuroprotective effects of rosuvastatin. It was shown that rosuvastatin decreased the expression of $\alpha$-synuclein and mTOR but increased the expression of Beclin-1 and AMPK pathways in the rotenone-induced neurotoxicity model in SH-SY5Y cells (an in vitro model of PD), leading to activation of autophagy [105]. Neuroinflammation-mediated neurodegeneration was alleviated through a combination of statins, including pravastatin, fluvastatin, simvastatin, pitavastatin, atorvastatin, and rosuvastatin [106]. Consequently, statins may modulate the autophagy pathways in the neurons and promote neuroprotective impressions in neurodegenerative diseases.

\section{Cardioprotective Effects of Statins}

Modulation of autophagy has been associated with cardioprotective effects in CVD [107]. Studies on the effects of 
TABLE 1: Modulation of autophagy by statins in different disorders.

\begin{tabular}{|c|c|c|c|c|}
\hline Disease type & Statin & Target cell/disease & Consequence & Reference \\
\hline \multirow{8}{*}{ Cancer } & Simvastatin & $\begin{array}{l}\text { Motoneuron-like NSC34 cells, } \\
\text { a model of neuroblastoma }\end{array}$ & $\begin{array}{l}\text { Reduction of autophagic vacuoles } \\
\text { and LC3II/I decreased cell survival }\end{array}$ & [103] \\
\hline & Simvastatin & Breast cancer cells of MDA-MB-231 & $\begin{array}{l}\text { Inhibition of autophagy } \\
\text { Reduced survival of breast cancer cells }\end{array}$ & {$[86]$} \\
\hline & Lovastatin & $\begin{array}{l}\text { Malignant mesothelioma ZL55 } \\
\text { cancer cells }\end{array}$ & $\begin{array}{c}\text { Stimulation of autophagy } \\
\text { Reduced survival of malignant } \\
\text { mesothelioma cells }\end{array}$ & {$[87]$} \\
\hline & Lovastatin & $\begin{array}{l}\text { Human malignant pleural } \\
\text { mesothelioma ACC-MESO1 cells }\end{array}$ & $\begin{array}{c}\text { Induction of autophagy } \\
\text { Decreased survival of malignant } \\
\text { pleural mesothelioma }\end{array}$ & {$[120]$} \\
\hline & Lovastatin & $\begin{array}{c}\text { Human malignant peripheral nerve } \\
\text { sheath tumor ST88-14 and NF90-8 } \\
\text { cells }\end{array}$ & $\begin{array}{l}\text { Perturbation of autophagy flux, } \\
\text { induction of nonapoptotic cell death } \\
\text { Decreased survival of malignant } \\
\text { peripheral nerve sheath tumor cells }\end{array}$ & [88] \\
\hline & Atorvastatin & Breast cancer MDA-MB-231 cells & $\begin{array}{l}\text { Stimulation of autophagy } \\
\text { Inhibition of breast cancer cell } \\
\text { proliferation }\end{array}$ & [92] \\
\hline & Pitavastatin & $\begin{array}{l}\text { Human melanoma WM115 and } \\
\text { A375 cells }\end{array}$ & $\begin{array}{l}\text { Induction of autophagy } \\
\text { Decreased survival of melanoma cells }\end{array}$ & [93] \\
\hline & Rosuvastatin & $\begin{array}{l}\text { Human papillary thyroid cancer } \\
\text { cells }\end{array}$ & $\begin{array}{c}\text { Suppression of autophagy } \\
\text { Induction of apoptosis in papillary } \\
\text { thyroid cancer cells }\end{array}$ & [94] \\
\hline Osteoarthritis & Simvastatin & Mouse model of OA & $\begin{array}{c}\text { Stimulation of autophagy } \\
\text { Deceleration of OA progression }\end{array}$ & [97] \\
\hline \multirow{4}{*}{$\begin{array}{l}\text { Cardiovascular } \\
\text { diseases }\end{array}$} & Pitavastatin & Cardiomyocyte & $\begin{array}{c}\text { Suppression of autophagy } \\
\text { Decreased cardiomyocyte injury }\end{array}$ & [109] \\
\hline & Simvastatin & Male Sprague-Dawley rat & $\begin{array}{c}\text { Suppression of autophagy } \\
\text { Decreased cardiomyocyte injury }\end{array}$ & [111] \\
\hline & Atorvastatin & Mouse model of atherosclerosis & $\begin{array}{c}\text { Stimulation of autophagy } \\
\text { Suppression of inflammation } \\
\text { Increased stability of vulnerable } \\
\text { atherosclerotic plaques }\end{array}$ & {$[108]$} \\
\hline & Rosuvastatin & Myocardial infarction model rat & $\begin{array}{c}\text { Induction of autophagy } \\
\text { Improved acute myocardial infarction }\end{array}$ & [110] \\
\hline \multirow[b]{2}{*}{$\begin{array}{l}\text { Neurodegenerative } \\
\text { diseases }\end{array}$} & Rosuvastatin & $\begin{array}{l}\text { Rotenone-induced neurotoxicity } \\
\text { model, PD SH-SY5Y cells }\end{array}$ & $\begin{array}{l}\text { Induction of autophagy } \\
\text { Neuroprotective function against PD }\end{array}$ & {$[105]$} \\
\hline & $\begin{array}{l}\text { Atorvastatin, pitavastatin, } \\
\text { fluvastatin, pravastatin, } \\
\text { simvastatin, and } \\
\text { rosuvastatin }\end{array}$ & $\begin{array}{c}\text { In vitro model of } \\
\text { lipopolysaccharide- (LPS-) } \\
\text { induced neuroinflammation }\end{array}$ & $\begin{array}{l}\text { Suppression of autophagy } \\
\text { Neuroprotective function }\end{array}$ & {$[106]$} \\
\hline
\end{tabular}

atorvastatin on the mouse model of vulnerable atherosclerotic plaque indicated that atorvastatin could promote the stability of vulnerable atherosclerotic plaques and reduced the lesions in the aorta. This was related to decreased lipid deposition as well as a reduced inflammatory state which was attributed to the inhibition of activation of nucleotidebinding oligomerization domain-like receptor pyrin domain containing 3 (NLRP3) inflammasome and decreased levels of inflammatory cytokines, such as tumor necrosis factor(TNF-) $\alpha$, IL-1 $\beta$, and IL-18. Furthermore, atorvastatin promoted the autophagy pathway in the cardiomyocyte by an mTOR-dependent approach [108]. Besides, pitavastatin prevented the autophagy pathway by downmodulating Beclin-1, resulting in a diminished cardiomyocyte injury [109]. Additionally, rosuvastatin was also associated with a cardioprotective activity that developed the autophagy pathway by promoting Beclin-1 expression and MAPK activation through inhibition of mTOR signaling [110]. Inhibition of AMPK to prevent autophagy in the cardiomyocytes resulted in abrogation of the cardioprotective function of rosuvastatin. Additionally, simvastatin was shown to suppressed autophagy signaling via reducing the LC3II/LC3I ratio and downregulating P62 expression and therefore conferred a cardioprotective effect [111]. This evidence implies the modulation of the autophagy pathways in the cardiomyocytes by statins, leading to protection against cardiac injuries. 


\section{Challenges of Statin Therapy}

Although several pros have been associated with statin therapy, some cons still exist. For instance, the bioavailability and the circulation of lipophilic statins upon oral intake are relatively low because of the first-pass metabolism by the liver and removal through the digestive process. The lower oral bioavailability may stem from weak solubility as well as low permeability. Pharmaceutical studies have been developing new strategies to overcome the lower oral bioavailability of statins [112]. Other than bioavailability, data have reported that statins may cause myopathies through different proposed mechanisms [113-115]. This effect might be selflimited myotoxicity through the direct effect of statins on the muscle and autoimmune responses by autoantibodies against 3-hydroxy-3-methylglutaryl coenzyme A reductase (HMGCR) [116]. Additionally, clinical trials have indicated that statin therapy might increase the risk of developing diabetes mellitus in prediabetic individuals. Approximately 10$20 \%$ out of 10,000 subjects receiving statin might develop diabetes annually [117]. As the rate of hemorrhagic stroke is increased when the cholesterol levels are reduced in the blood, patients with statin therapy may exercise caution [118]. Even though statin therapy has been associated with increased liver enzymes, hepatotoxicity might be rarely seen [119]. Regarding the disadvantages of statin therapy (even if rare), patients with specific considerations should be evaluated before initiating the treatment regimen.

\section{Conclusions and Future Directions}

A bulk of investigations have indicated the ameliorative effects of statins on treating a wide range of disorders (Table 1). The therapeutic properties of statins have been attributed to the modulation of various molecules involved in the autophagy pathways. Autophagy is a mechanism of the cells under stress to promote survival and the resolution of the damaged and superfluous organelles within the cells. Considering the vital role of autophagy in maintaining healthy conditions and the importance of autophagy dysregulation in the etiopathogenesis of several disorders, modulation of the autophagy signaling pathway might confer a promising therapeutic approach. As a well-known statin, simvastatin has been associated with cardioprotective and neuroprotective properties. Furthermore, simvastatin therapy reduces the cancerous behaviors of tumor cells and has been shown to decelerate the progression of OA. Another statin, lovastatin, promotes autophagy and inhibits the proliferation and migration of tumor cells. Additionally, induction of autophagy has been attributed to pitavastatin therapy that reduces the survival of melanoma tumor cells. However, it has been associated with the suppression of autophagy and cardioprotective characteristics. Another statin, fluvastatin, has been shown to induce autophagy and hence exert antitumor properties. Taken together, it seems that modulation of autophagy by statins has promising potential in the protection of neurons and myocytes as well as in decreasing adverse cancerous behaviors of tumor cells and degradation of cartilages in OA patients. That notwith- standing, further experimental and clinical trials are needed to clarify the therapeutic characteristics of statins and safety and toxicity issues.

\section{Data Availability}

There is no raw data associated with this review.

\section{Conflicts of Interest}

The authors declare that they have no conflicts of interest.

\section{Authors' Contributions}

Armita Mahdavi Gorabi and Nasim Kiaie equally contributed as the first authors.

\section{References}

[1] M. Noguchi, N. Hirata, T. Tanaka, F. Suizu, H. Nakajima, and J. A. Chiorini, "Autophagy as a modulator of cell death machinery," Cell Death \& Disease, vol. 11, no. 7, pp. 512517,2020 .

[2] I. Tanida, T. Ueno, and E. Kominami, "LC3 and autophagy," in Autophagosome and phagosome, pp. 77-88, Springer, 2008.

[3] B. Levine and G. Kroemer, "Autophagy in the pathogenesis of disease," Cell, vol. 132, no. 1, pp. 27-42, 2008.

[4] J. D. Rabinowitz and E. White, "Autophagy and metabolism," Science, vol. 330, no. 6009, pp. 1344-1348, 2010.

[5] S. A. Gudipaty, C. M. Conner, J. Rosenblatt, and D. J. Montell, "Unconventional ways to live and die: cell death and survival in development, homeostasis, and disease," Annual Review of Cell and Developmental Biology, vol. 34, pp. 311332, 2018.

[6] A. KELEKAR, "Autophagy," Annals of the New York Academy of Sciences, vol. 1066, no. 1, pp. 259-271, 2005.

[7] D. J. Wu and I. E. Adamopoulos, "Autophagy and autoimmunity," Clinical Immunology, vol. 176, pp. 55-62, 2017.

[8] B. Levine, "Autophagy and cancer," Nature, vol. 446, no. 7137, pp. 745-747, 2007.

[9] R. A. Nixon, "The role of autophagy in neurodegenerative disease," Nature Medicine, vol. 19, no. 8, pp. 983-997, 2013.

[10] R. L. Vidal, S. Matus, L. Bargsted, and C. Hetz, "Targeting autophagy in neurodegenerative diseases," Trends in Pharmacological Sciences, vol. 35, no. 11, pp. 583-591, 2014.

[11] N. Tilija Pun, W. J. Jang, and C. H. Jeong, "Role of autophagy in regulation of cancer cell death/apoptosis during anticancer therapy: focus on autophagy flux blockade," Archives of Pharmacal Research, vol. 43, no. 5, pp. 475-488, 2020.

[12] J. Wang and G. S. Wu, "Role of Autophagy in Cisplatin Resistance in Ovarian Cancer Cells," Journal of Biological Chemistry, vol. 289, no. 24, pp. 17163-17173, 2014.

[13] D. Kong, S. Ma, B. Liang et al., "The different regulatory effects of p53 status on multidrug resistance are determined by autophagy in ovarian cancer cells," Biomedicine \& Pharmacotherapy, vol. 66, no. 4, pp. 271-278, 2012.

[14] Q. Zhang, S. Si, S. Schoen, J. Chen, X. B. Jin, and G. Wu, “Suppression of autophagy enhances preferential toxicity of paclitaxel to folliculin-deficient renal cancer cells," Journal of Experimental \& Clinical Cancer Research, vol. 32, no. 1, p. 99, 2013. 
[15] Q. Li, Y. Liu, and M. Sun, “Autophagy and Alzheimer's disease," Cellular and Molecular Neurobiology, vol. 37, no. 3, pp. 377-388, 2017.

[16] A. Scrivo, M. Bourdenx, O. Pampliega, and A. M. Cuervo, "Selective autophagy as a potential therapeutic target for neurodegenerative disorders," The Lancet Neurology, vol. 17, no. 9, pp. 802-815, 2018.

[17] O. Sizar, S. Khare, R. T. Jamil, and R. Talati, Statin medications, in StatPearls, StatPearls Publishing, Treasure Island (FL), 2021, https://www.ncbi.nlm.nih.gov/books/ NBK430940/.

[18] U. Galicia-Garcia, S. Jebari, A. Larrea-Sebal et al., "Statin treatment-induced development of type 2 diabetes: from clinical evidence to mechanistic insights," International Journal of Molecular Sciences, vol. 21, no. 13, p. 4725, 2020.

[19] A. R. Afshari, H. Mollazadeh, N. C. Henney, T. Jamialahmad, and A. Sahebkar, "Effects of statins on brain tumors: a review," Seminars in Cancer Biology, vol. 73, pp. 116-133, 2021.

[20] M. Alidadi, F. Montecucco, T. Jamialahmadi, K. al-Rasadi, T. P. Johnston, and A. Sahebkar, "Beneficial effect of statin therapy on arterial stiffness," BioMed Research International, vol. 2021, Article ID 5548310, 19 pages, 2021.

[21] A. Bahrami, N. Parsamanesh, S. L. Atkin, M. Banach, and A. Sahebkar, "Effect of statins on toll-like receptors: a new insight to pleiotropic effects," Pharmacological Research, vol. 135, pp. 230-238, 2018.

[22] A. M. Gorabi, N. Kiaie, M. Pirro, V. Bianconi, T. Jamialahmadi, and A. Sahebkar, "Effects of statins on the biological features of mesenchymal stem cells and therapeutic implications," Heart Failure Reviews, vol. 26, no. 5, pp. 12591272, 2021.

[23] H. Mollazadeh, E. Tavana, G. Fanni et al., "Effects of statins on mitochondrial pathways," Journal of Cachexia, Sarcopenia and Muscle, vol. 12, no. 2, pp. 237-251, 2021.

[24] Ž. Reiner, M. Hatamipour, M. Banach et al., "Statins and the Covid-19 main protease: in silico evidence on direct interaction," Archives of Medical Science, vol. 16, no. 3, pp. 490-496, 2020.

[25] A. Sahebkar, C. Serban, S. Ursoniu et al., "The impact of statin therapy on plasma levels of von Willebrand factor antigen," Thrombosis and Haemostasis, vol. 115, no. 3, pp. 520532, 2016.

[26] A. M. Gorabi, N. Kiaie, V. Bianconi et al., "Antiviral effects of statins," Progress in Lipid Research, vol. 79, p. 101054, 2020.

[27] S. M. Jeong, W. Jang, and D. W. Shin, "Association of statin use with Parkinson's disease: dose-response relationship," Movement Disorders, vol. 34, no. 7, pp. 1014-1021, 2019.

[28] J. L. Schultz, P. C. Nopoulos, A. Killoran, and J. A. Kamholz, "Statin use and delayed onset of Huntington's disease," Movement Disorders, vol. 34, no. 2, pp. 281-285, 2019.

[29] H. Nakatogawa, K. Suzuki, Y. Kamada, and Y. Ohsumi, "Dynamics and diversity in autophagy mechanisms: lessons from yeast," Nature Reviews Molecular Cell Biology, vol. 10, no. 7, pp. 458-467, 2009.

[30] A. Parkhitko, O. Favorova, and E. Henske, "Autophagy: mechanisms, regulation, and its role in tumorigenesis," Biochemistry, vol. 78, no. 4, pp. 355-367, 2013.

[31] C. Settembre, "Transcriptional regulation of autophagy: mechanisms and diseases," Frontiers in cell and developmental biology, vol. 7, p. 114, 2019.
[32] G. Marino and C. López-Otín, “Autophagy: molecular mechanisms, physiological functions and relevance in human pathology," Cellular and Molecular Life Sciences CMLS, vol. 61, no. 12, pp. 1439-1454, 2004.

[33] N. Mizushima, "Autophagy: process and function," Genes \& Development, vol. 21, no. 22, pp. 2861-2873, 2007.

[34] Y. Feng, D. He, Z. Yao, and D. J. Klionsky, "The machinery of macroautophagy," Cell Research, vol. 24, no. 1, pp. 24-41, 2014.

[35] C. Münz, “Autophagy in immunity," Progress in Molecular Biology and Translational Science, vol. 172, pp. 67-85, 2020.

[36] W. W. Li, J. Li, and J. K. Bao, "Microautophagy: lesser-known self-eating," Cellular and Molecular Life Sciences, vol. 69, no. 7, pp. 1125-1136, 2012.

[37] S. Kaushik and A. M. Cuervo, "Chaperone-mediated autophagy: a unique way to enter the lysosome world," Trends in Cell Biology, vol. 22, no. 8, pp. 407-417, 2012.

[38] V. Kaminskyy and B. Zhivotovsky, "Proteases in autophagy," Biochimica et Biophysica Acta (BBA)-Proteins and Proteomics, vol. 1824, no. 1, pp. 44-50, 2012.

[39] P. Jiang and N. Mizushima, "Autophagy and human diseases,” Cell Research, vol. 24, no. 1, pp. 69-79, 2014.

[40] A. M. Choi, S. W. Ryter, and B. Levine, "Autophagy in human health and disease," New England Journal of Medicine, vol. 368, no. 7, pp. 651-662, 2013.

[41] D. Glick, S. Barth, and K. F. Macleod, "Autophagy: cellular and molecular mechanisms," The Journal of Pathology, vol. 221, no. 1, pp. 3-12, 2010.

[42] K. R. Parzych and D. J. Klionsky, "An overview of autophagy: morphology, mechanism, and regulation," Antioxidants \& Redox Signaling, vol. 20, no. 3, pp. 460-473, 2014.

[43] S. Sforzini, M. N. Moore, C. Oliveri et al., "Role of mTOR in autophagic and lysosomal reactions to environmental stressors in molluscs," Aquatic Toxicology, vol. 195, pp. 114-128, 2018.

[44] C. H. Jung, S. H. Ro, J. Cao, N. M. Otto, and D. H. Kim, "mTOR regulation of autophagy," FEBS Letters, vol. 584, no. 7, pp. 1287-1295, 2010.

[45] S. Alers, A. S. Löffler, F. Paasch et al., "Atg13 and FIP200 act independently of Ulk1 and Ulk2 in autophagy induction," Autophagy, vol. 7, no. 12, pp. 1424-1433, 2011.

[46] Z. Yue, S. Jin, C. Yang, A. J. Levine, and N. Heintz, "Beclin 1, an autophagy gene essential for early embryonic development, is a haploinsufficient tumor suppressor," Proceedings of the National Academy of Sciences, vol. 100, no. 25, pp. 15077-15082, 2003.

[47] J. Wang, "Beclin 1 bridges autophagy, apoptosis and differentiation," Autophagy, vol. 4, no. 7, pp. 947-948, 2008.

[48] D. G. Hardie, "AMPK and autophagy get connected," The EMBO Journal, vol. 30, no. 4, pp. 634-635, 2011.

[49] S. Alers, A. S. Löffler, S. Wesselborg, and B. Stork, "Role of AMPK-mTOR-Ulk1/2 in the regulation of autophagy: cross talk, shortcuts, and feedbacks," Molecular and Cellular Biology, vol. 32, no. 1, pp. 2-11, 2012.

[50] D. J. Klionsky, J. M. Cregg, W. A. Dunn Jr. et al., "A unified nomenclature for yeast autophagy-related genes," Developmental Cell, vol. 5, no. 4, pp. 539-545, 2003.

[51] S. R. Yoshii and N. Mizushima, "Autophagy machinery in the context of mammalian mitophagy," Biochimica Et Biophysica Acta (BBA)-Molecular Cell Research, vol. 1853, no. 10, pp. 2797-2801, 2015. 
[52] Q. Wang and J. Ren, "mTOR-independent autophagy inducer trehalose rescues against insulin resistance-induced myocardial contractile anomalies: role of p38 MAPK and Foxo1," Pharmacological Research, vol. 111, pp. 357-373, 2016.

[53] M. Zhou, W. Xu, J. Wang et al., "Boosting mTOR-dependent autophagy via upstream TLR4-MyD88-MAPK signalling and downstream NF- $\kappa$ B pathway quenches intestinal inflammation and oxidative stress injury," eBioMedicine, vol. 35, pp. 345-360, 2018.

[54] D. J. Maron, S. Fazio, and M. F. Linton, "Current perspectives on statins," Circulation, vol. 101, no. 2, pp. 207-213, 2000.

[55] S. E. Nissen, E. M. Tuzcu, P. Schoenhagen et al., "Statin therapy, LDL cholesterol, C-reactive protein, and coronary artery disease," New England Journal of Medicine, vol. 352, no. 1, pp. 29-38, 2005.

[56] A. Endo, "The origin of the statins," in International Congress Series, Elsevier, 2004.

[57] E. S. Istvan and J. Deisenhofer, "Structural mechanism for statin inhibition of HMG-CoA reductase," Science, vol. 292, no. 5519, pp. 1160-1164, 2001.

[58] I. Gouni-Berthold, H. K. Berthold, H. Gylling et al., "Effects of ezetimibe and/or simvastatin on LDL receptor protein expression and on LDL receptor and HMG-CoA reductase gene expression: a randomized trial in healthy men," Atherosclerosis, vol. 198, no. 1, pp. 198-207, 2008.

[59] A. Endo, M. Kuroda, and Y. Tsujita, "ML-236A, ML-236B, and ML-236C, new inhibitors of cholesterogensis produced by Penicillium citrinum," The Journal of Antibiotics, vol. 29, no. 12, pp. 1346-1348, 1976.

[60] A. Alberts, J. Chen, G. Kuron et al., "Mevinolin: a highly potent competitive inhibitor of hydroxymethylglutarylcoenzyme A reductase and a cholesterol-lowering agent," Proceedings of the National Academy of Sciences, vol. 77, no. 7, pp. 3957-3961, 1980.

[61] M. Schonewille, J. Freark de Boer, L. Mele et al., "Statins increase hepatic cholesterol synthesis and stimulate fecal cholesterol elimination in mice," Journal of Lipid Research, vol. 57, no. 8, pp. 1455-1464, 2016.

[62] C. Laezza, A. M. Malfitano, M. C. Proto et al., "Inhibition of 3-hydroxy-3-methylglutaryl-coenzyme A reductase activity and of Ras farnesylation mediate antitumor effects of anandamide in human breast cancer cells," Endocrine-Related Cancer, vol. 17, no. 2, pp. 495-503, 2010.

[63] A. Szkopińska and D. Płochocka, "Farnesyl diphosphate synthase; regulation of product specificity," Acta Biochimica Polonica, vol. 52, no. 1, pp. 45-55, 2005.

[64] K. Okada, T. Saito, T. Nakagawa, M. Kawamukai, and Y. Kamiya, "Five geranylgeranyl diphosphate synthases expressed in different organs are localized into three subcellular compartments in Arabidopsis," Plant Physiology, vol. 122, no. 4, pp. 1045-1056, 2000.

[65] S. S. Agabiti, Y. Liang, and A. J. Wiemer, "Molecular mechanisms linking geranylgeranyl diphosphate synthase to cell survival and proliferation," Molecular Membrane Biology, vol. 33, no. 1-2, pp. 1-11, 2016.

[66] Y. Rikitake and J. K. Liao, "Rho GTPases, statins, and nitric oxide," Circulation Research, vol. 97, no. 12, pp. 1232-1235, 2005.

[67] U. Rauch, J. I. Osende, J. H. Chesebro et al., "Statins and cardiovascular diseases: the multiple effects of lipid-lowering therapy by statins," Atherosclerosis, vol. 153, no. 1, pp. 181$189,2000$.

[68] Q. Zhou and J. K. Liao, "Statins and cardiovascular diseases: from cholesterol lowering to pleiotropy," Current Pharmaceutical Design, vol. 15, no. 5, pp. 467-478, 2009.

[69] E. S. Stroes, P. D. Thompson, A. Corsini et al., "Statin-associated muscle symptoms: impact on statin therapy-European Atherosclerosis Society consensus panel statement on assessment, aetiology and management," European Heart Journal, vol. 36, no. 17, pp. 1012-1022, 2015.

[70] D.-W. Kim, D. H. Kim, J. H. Park et al., “Association between statin treatment and new-onset diabetes mellitus: a population based case-control study," Diabetology \& Metabolic Syndrome, vol. 11, no. 1, pp. 1-8, 2019.

[71] M. J. Ko, A. J. Jo, Y. J. Kim et al., “Time- and dose-dependent association of statin use with risk of clinically relevant newonset diabetes mellitus in primary prevention: a nationwide observational cohort study," Journal of the American Heart Association, vol. 8, no. 8, p. e011320, 2019.

[72] P. Thavendiranathan, A. Bagai, M. A. Brookhart, and N. K. Choudhry, "Primary prevention of cardiovascular diseases with statin therapy: a meta-analysis of randomized controlled trials," Archives of Internal Medicine, vol. 166, no. 21, pp. 2307-2313, 2006.

[73] Y. Chen, B. Feng, and Z. Chen, "Statins for primary prevention of cardiovascular and cerebrovascular events in diabetic patients without established cardiovascular diseases: a metaanalysis," Experimental and Clinical Endocrinology \& Diabetes, vol. 120, no. 2, pp. 116-120, 2012.

[74] X.-C. Bao et al., "Simvastatin decreases hyperbaric oxygeninduced acute lung injury by upregulating eNOS," American Journal of Physiology-Lung Cellular and Molecular Physiology, vol. 314, no. 2, pp. L287-L297, 2018.

[75] C. P. Barbosa, L. Bracht, F. Q. Ames, F. M. de Souza SilvaComar, R. P. Tronco, and C. A. Bersani-Amado, "Effects of ezetimibe, simvastatin, and their combination on inflammatory parameters in a rat model of adjuvant-induced arthritis," Inflammation, vol. 40, no. 2, pp. 717-724, 2017.

[76] G. Rodrigues, A. J. Moreira, S. Bona et al., "Simvastatin reduces hepatic oxidative stress and endoplasmic reticulum stress in nonalcoholic steatohepatitis experimental model," Oxidative Medicine and Cellular Longevity, vol. 2019, 10 pages, 2019.

[77] C. Zhang, X. M. Ren, H. B. Li et al., "Simvastatin alleviates inflammation and oxidative stress in rats with cerebral hemorrhage through Nrf2-ARE signaling pathway," European Review for Medical and Pharmacological Sciences, vol. 23, no. 14, pp. 6321-6329, 2019.

[78] S. Dhakal, M. Subhan, J. M. Fraser, K. Gardiner, and I. Macreadie, "Simvastatin efficiently reduces levels of Alzheimer's amyloid beta in yeast," International Journal of Molecular Sciences, vol. 20, no. 14, p. 3531, 2019.

[79] J. Yan, L. Qiao, J. Wu, H. Fan, J. Sun, and Y. Zhang, "Simvastatin protects dopaminergic neurons against MPP+-induced oxidative stress and regulates the endogenous anti-oxidant system through ERK," Cellular Physiology and Biochemistry, vol. 51, no. 4, pp. 1957-1968, 2018.

[80] C. P. Lau, C. S. H. Fung, K. C. Wong et al., "Simvastatin possesses antitumor and differentiation-promoting properties that affect stromal cells in giant cell tumor of bone," Journal of Orthopaedic Research, vol. 38, no. 2, pp. 297-310, 2020. 
[81] H. C. Hendrie, MBChB, DSc, A. Hake, MD, K. Lane, MS et al., "Statin use, incident dementia and Alzheimer disease in elderly African Americans," Ethnicity \& Disease, vol. 25, no. 3, pp. 345-354, 2015.

[82] A. Gupta, W. Stokes, M. Eguchi et al., "Statin use associated with improved overall and cancer specific survival in patients with head and neck cancer," Oral Oncology, vol. 90, pp. 5466, 2019.

[83] J. Bouitbir, G. M. Sanvee, M. V. Panajatovic, F. Singh, and S. Krähenbühl, "Mechanisms of statin-associated skeletal muscle-associated symptoms," Pharmacological Research, vol. 154, p. 104201, 2020.

[84] S. Korani, M. Korani, S. Bahrami et al., "Application of nanotechnology to improve the therapeutic benefits of statins," Drug Discovery Today, vol. 24, no. 2, pp. 567-574, 2019.

[85] C. Mattiuzzi and G. Lippi, "Current cancer epidemiology," Journal of epidemiology and global health, vol. 9, no. 4, pp. 217-222, 2019.

[86] Y. C. Castellanos-Esparza, S. Wu, L. Huang et al., "Synergistic promoting effects of pentoxifylline and simvastatin on the apoptosis of triple-negative MDA-MB-231 breast cancer cells," International Journal of Oncology, vol. 52, no. 4, pp. 1246-1254, 2018.

[87] Y. Shi, E. Felley-Bosco, T. M. Marti, and R. A. Stahel, "Differential effects of lovastatin on cisplatin responses in normal human mesothelial cells versus cancer cells: implication for therapy," PLoS One, vol. 7, no. 9, article e45354, 2012.

[88] J. W. Wojtkowiak, K. M. Sane, M. Kleinman, B. F. Sloane, J. J. Reiners Jr., and R. R. Mattingly, "Aborted autophagy and nonapoptotic death induced by farnesyl transferase inhibitor and lovastatin," Journal of Pharmacology and Experimental Therapeutics, vol. 337, no. 1, pp. 65-74, 2011.

[89] K. A. Manu, M. K. Shanmugam, F. Li et al., "Simvastatin sensitizes human gastric cancer xenograft in nude mice to capecitabine by suppressing nuclear factor-kappa B-regulated gene products," Journal of Molecular Medicine (Berlin, Germany), vol. 92, no. 3, pp. 267-276, 2014.

[90] P. Carmeliet and R. K. Jain, "Angiogenesis in cancer and other diseases," nature, vol. 407, no. 6801, pp. 249-257, 2000.

[91] S. Quintero-Fabián, R. Arreola, E. Becerril-Villanueva et al., "Role of matrix metalloproteinases in angiogenesis and cancer," Frontiers in Oncology, vol. 9, p. 1370, 2019.

[92] M.-B. Hu, J. W. Zhang, J. B. Gao et al., "Atorvastatin induces autophagy in MDA-MB-231 breast cancer cells," Ultrastructural Pathology, vol. 42, no. 5, pp. 409-415, 2018.

[93] A. Al-Qatati and S. Aliwaini, "Combined pitavastatin and dacarbazine treatment activates apoptosis and autophagy resulting in synergistic cytotoxicity in melanoma cells," Oncology Letters, vol. 14, no. 6, pp. 7993-7999, 2017.

[94] N. D. Zeybek, N. E. Gulcelik, F. F. Kaymaz et al., "Rosuvastatin induces apoptosis in cultured human papillary thyroid cancer cells," Journal of Endocrinology, vol. 210, no. 1, p. 105, 2011.

[95] Z. Yang, Z. Su, J. P. DeWitt et al., "Fluvastatin prevents lung adenocarcinoma bone metastasis by triggering autophagy," eBioMedicine, vol. 19, pp. 49-59, 2017.

[96] Y. He, Z. Li, P. G. Alexander et al., "Pathogenesis of osteoarthritis: risk factors, regulatory pathways in chondrocytes, and experimental models," Biology, vol. 9, no. 8, p. 194, 2020.

[97] T. Tanaka, T. Matsushita, K. Nishida et al., "Attenuation of osteoarthritis progression in mice following intra-articular administration of simvastatin-conjugated gelatin hydrogel," Journal of Tissue Engineering and Regenerative Medicine, vol. 13, no. 3, pp. 423-432, 2019.

[98] Z. Mao, P. Wang, Q. Pan et al., "Pravastatin alleviates interleukin $1 \beta$-induced cartilage degradation by restoring impaired autophagy associated with MAPK pathway inhibition," International Immunopharmacology, vol. 64, pp. 308318, 2018.

[99] Y. Liao, P. Zhang, B. Yuan, L. Li, and S. Bao, "Pravastatin protects against avascular necrosis of femoral head via autophagy," Frontiers in Physiology, vol. 9, p. 307, 2018.

[100] J. Wang, J. Dong, J. Yang, Y. Wang, and J. Liu, “Association between statin use and incidence or progression of osteoarthritis: meta-analysis of observational studies," Osteoarthritis and Cartilage, vol. 28, no. 9, pp. 1170-1179, 2020.

[101] M. Zatyka, S. Sarkar, and T. Barrett, "Autophagy in Rare (NonLysosomal) Neurodegenerative Diseases," Journal of Molecular Biology, vol. 432, no. 8, pp. 2735-2753, 2020.

[102] Y. Yang and D. J. Klionsky, "Autophagy and disease: unanswered questions," Cell Death \& Differentiation, vol. 27, no. 3, pp. 858-871, 2020.

[103] W. Qi, L. Yan, Y. Liu et al., "Simvastatin aggravates impaired autophagic flux in NSC34-hSOD1G93A cells through inhibition of geranylgeranyl pyrophosphate synthesis," Neuroscience, vol. 409, pp. 130-141, 2019.

[104] Y. Liu, H. N. Yang, G. Y. Jia et al., "The synergistic neuroprotective effects of combined rosuvastatin and resveratrol pretreatment against cerebral ischemia/reperfusion injury," Journal of Stroke and Cerebrovascular Diseases, vol. 27, no. 6, pp. 1697-1704, 2018.

[105] S. Y. Kang, S. B. Lee, H. J. Kim, H. T. Kim, H. O. Yang, and W. Jang, "Autophagic modulation by rosuvastatin prevents rotenone-induced neurotoxicity in an in vitro model of Parkinson's disease," Neuroscience Letters, vol. 642, pp. 20-26, 2017.

[106] A. McFarland, A. K. Davey, C. M. McDermott, G. D. Grant, J. Lewohl, and S. Anoopkumar-Dukie, "Differences in statin associated neuroprotection corresponds with either decreased production of IL- $1 \beta$ or TNF- $\alpha$ in an _in vitro model of neuroinflammation-induced neurodegeneration," Toxicology and Applied Pharmacology, vol. 344, pp. 56-73, 2018.

[107] A. Ludman, V. Venugopal, D. M. Yellon, and D. J. Hausenloy, "Statins and cardioprotection - More than just lipid lowering?," Pharmacology \& Therapeutics, vol. 122, no. 1, pp. 3043, 2009.

[108] S. Peng, L. W. Xu, X. Y. Che et al., "Atorvastatin inhibits inflammatory response, attenuates lipid deposition, and improves the stability of vulnerable atherosclerotic plaques by modulating autophagy," Frontiers in Pharmacology, vol. 9, p. 438, 2018.

[109] Z. Zha, J. H. Wang, S. L. Li, and Y. Guo, "Pitavastatin attenuates AGEs-induced mitophagy via inhibition of ROS generation in the mitochondria of cardiomyocytes," Journal of Biomedical Research, vol. 32, no. 4, pp. 281-287, 2018.

[110] Z. Song, L. Chen, D. Zhang, S. Y. Zhang, and X. Lin, "Rosuvastatin protects acute myocardial infarction rats through autophagy regulation via AMPK signaling," Zhonghua Yi Xue Za Zhi, vol. 98, no. 43, pp. 3536-3541, 2018.

[111] F. Su, M. Shi, J. Zhang et al., "Simvastatin protects heart from pressure overload injury by inhibiting excessive autophagy," 
International Journal of Medical Sciences, vol. 15, no. 13, pp. 1508-1516, 2018.

[112] T. R. Meola, H. B. Schultz, K. F. Peressin, and C. A. Prestidge, "Enhancing the oral bioavailability of simvastatin with silicalipid hybrid particles: the effect of supersaturation and silica geometry," European Journal of Pharmaceutical Sciences, vol. 150, p. 105357, 2020.

[113] A. Selva-O'Callaghan, M. Alvarado-Cardenas, I. Pinal-Fernández et al., "Statin-induced myalgia and myositis: an update on pathogenesis and clinical recommendations," Expert Review of Clinical Immunology, vol. 14, no. 3, pp. 215-224, 2018.

[114] N. C. Ward, G. F. Watts, and R. H. Eckel, "Statin toxicity," Circulation Research, vol. 124, no. 2, pp. 328-350, 2019.

[115] M. Banach, C. Serban, S. Ursoniu et al., "Statin therapy and plasma coenzyme Q10 concentrations-a systematic review and meta-analysis of placebo-controlled trials," Pharmacological Research, vol. 99, pp. 329-336, 2015.

[116] S. Jayatilaka, K. Desai, S. Rijal, and D. Zimmerman, "Statininduced autoimmune necrotizing myopathy," Journal of Primary Care and Community Health, vol. 12, p. 215013272110287, 2021.

[117] N. Sattar, D. Preiss, H. M. Murray et al., "Statins and risk of incident diabetes: a collaborative meta-analysis of randomised statin trials," Lancet, vol. 375, no. 9716, pp. 735-742, 2010.

[118] B. E. Sanz-Cuesta and J. L. Saver, "Lipid-lowering therapy and hemorrhagic stroke risk: comparative meta-analysis of statins and PCSK9 inhibitors," Stroke, 2021.

[119] E. Björnsson, E. I. Jacobsen, and E. Kalaitzakis, "Hepatotoxicity associated with statins: reports of idiosyncratic liver injury post-marketing," Journal of Hepatology, vol. 56, no. 2, pp. 374-380, 2012.

[120] K. Asakura, Y. Izumi, M. Yamamoto et al., "The cytostatic effects of lovastatin on ACC-MESO-1 cells," Journal of Surgical Research, vol. 170, no. 2, pp. e197-e209, 2011. 